\title{
Polyphyllin I inhibits invasion and epithelial-mesenchymal transition via CIP2A/PP2A/ERK signaling in prostate cancer
}

\author{
XUEWEN LIU ${ }^{1,2^{*}}$, ZHITING SUN ${ }^{2 *}$, JIKUN DENG $^{1}$, JUN LIU $^{1}$, KAIHUAI MA ${ }^{1}$, \\ YUAN SI ${ }^{1,2}$, TE ZHANG ${ }^{2}$, TINGTING FENG ${ }^{3}$, YING LIU ${ }^{1,2}$ and YAN TAN ${ }^{1,4}$ \\ ${ }^{1}$ School of Basic Sciences, Hubei University of Medicine; ${ }^{2}$ Laboratory of Molecular Target Therapy of Cancer, \\ Institute of Basic Medical Sciences, Hubei University of Medicine, Shiyan, Hubei 442000; \\ ${ }^{3}$ School of Basic Medical Sciences, Anhui Medical University, Hefei, Anhui 230032; \\ ${ }^{4}$ Deparment of Andrology, Renmin Hospital, Hubei University of Medicine, Shiyan, Hubei 442000, P.R. China
}

Received January 19, 2018; Accepted June 1, 2018

DOI: $10.3892 /$ ijo.2018.4464

\begin{abstract}
Polyphyllin I (PPI) is a natural compound extracted from the rhizomes of Paris polyphylla and has been used to treat fevers and headaches in China. In the present study, the antitumor activity of PPI in prostate cancer (PC) cells was evaluated. At low doses, PPI decreased proliferation, invasion and epithelial-mesenchymal transition (EMT) in PC cells. PPI decreased the expression of matrix metalloproteinase 7 (MMP7), an enzyme that is critical for tumor metastasis. PPI also decreased the expression of Snail and vimentin, which are EMT-associated factors. Additionally, PPI suppressed AP-1 transcriptional activity and AP-1 binding to the MMP7 and vimentin promoters. The results demonstrated that PPI downregulated the phosphorylation of extracellular signaling-related kinase (ERK), which is upstream modulator of AP-1. The results of the present study demonstrated that PPI may inhibit the cancerous inhibitor of protein phosphatase 2A (CIP2A)/protein phosphatase 2A (PP2A)/ERK axis, downregulate the expression of MMP7, vimentin, and Snail, and suppress tumor invasion and EMT. A PC xenograft mouse model was employed and the results revealed that PPI may decrease tumor growth and weight. Additionally, PPI may inhibit proliferating cell nuclear antigen expression and
\end{abstract}

Correspondence to: Professor Yan Tan, Department of Andrology, Renmin Hospital, Hubei University of Medicine, Shiyan, Hubei 442000, P.R. China

E-mail: tanyan-1@163.com

Dr Ying Liu, Laboratory of Molecular Target Therapy of Cancer, Institute of Basic Medical Sciences; Hubei University of Medicine, Shiyan, Hubei 442000, P.R. China

E-mail: ying_liu1002@163.com

*Contributed equally

Key words: polyphyllin I, prostate cancer, cancerous inhibitor of protein phosphatase $2 \mathrm{~A}$, metastasis, matrix metalloproteinase 7 , epithelial-mesenchymal transition
CIP2A/PP2A/ERK signaling pathway in PPI-treated tumors. Therefore, the results of the present study suggest that PPI may suppress the growth, invasion and EMT of PC cells via inhibition of CIP2A/PP2A/ERK signaling axis. As a result, PPI may be a novel target for the treatment of PC.

\section{Introduction}

Prostate cancer (PC) is a common malignancy diagnosed in men. In 2012, a total of $\sim 1,1111,700$ cases of PC were newly diagnosed, and 307,500 mortality cases have been estimated worldwide (1). The morbidity of PC is increasing in China in recent years (2). Although patients diagnosed with localized PC exhibit a survival rate of $>5$ years, the majority of patients with PC will progress to castration-resistant PC, and eventually, relapse (3). Therefore, novel drugs that may suppress the tumor initiation, progression and metastasis of $\mathrm{PC}$ are required.

Oncogenes and anti-oncogenes serve pivotal roles in the pathogenesis of several cancer types. Cancerous inhibitor of protein phosphatase 2A (CIP2A) is an oncoprotein that suppresses protein phosphatase $2 \mathrm{~A}$ (PP2A) activity in the degradation of c-Myc protein in cancer cells (4). CIP2A is overexpressed in numerous types of human cancer, including PC, gastric, colon, breast, head and neck, lung and ovarian cancer (4-10). Additionally, CIP2A overexpression is associated with malignant proliferation and aggressiveness (11). De et al suggested a role of oncogenic nexus of CIP2A in carcinogenesis (12). This nexus is created by CIP2A-mediated inhibition of PP2A, which activates oncogenic signal factors including Akt, extracellular signaling-related kinase (ERK) and c-Myc, and thus may serve as a biomarker and therapeutic target for the treatment of cancer. Recently, Wu et al reported that CIP2A physically associates with H-Ras and activates the MEK/ERK signaling pathway, thus promoting epithelialmesenchymal transition (EMT) and progression of cervical cancer (13). Therefore, targeting CIP2A may be a promising therapeutic strategy for cancer.

Increasing evidences has shown that natural products not only serve key roles in the detection and evolvement of novel drugs, but they may be used as molecular probes for screening treatment targets $(14,15)$. Paris polyphyllin is a 
medicinal herb in the Shennongiia National Nature Reserve of China, which may treat fevers, headaches, burns, wounds, and exhibit immune-regulatory and antitumor abilities $(16,17)$. Polyphyllin I (PPI), a bioactive phytochemical isolated from the rhizoma of $P$. polyphylla, exhibits preclinical antitumor efficacy in several cancer types, including breast, lung, gastric, and ovarian cancer, as well as osteosarcoma $(16,18,19)$. Recently, PPI was found to increase the sensitivity of liver cancer HepG2 cells to cisplatin, and activate autophagy via the PI3K/Akt/mTOR signaling pathway (20). The antitumor activity of PPI on PC cells has not previously been evaluated. The aim of the present study is to evaluate the antitumor effects and potential molecular mechanisms of PPI in PC.

\section{Materials and methods}

Reagents. PPI with a purity of $98 \%$ was purchased from Shanghai YuanYe Bio-Technology Co., Ltd. (Shanghai, China). PPI was dissolved in dimethylsulfoxide (DMSO; Sigma-Aldrich; Merck KGaA, Darmstadt, Germany) at a stock solution of $4 \mathrm{mM}$ and stored at $-20^{\circ} \mathrm{C}$. Okadaic acid (OA) was purchased from Sigma-Aldrich; Merck KGaA.

Cell culture. Human PC cancer cell lines PC 3 and DU145 were purchased from American Type Culture Collection (ATCC; Manassas, VA, USA) and cultured in Dulbecco's modified Eagle's medium (DMEM; high glucose; Gibco; Thermo Fisher Scientific, Inc., Waltham, MA, USA) containing $10 \%$ fetal bovine serum (FBS; HyClone, Logan, UT, USA), $100 \mathrm{U} / \mathrm{ml}$ penicillin and $100 \mu \mathrm{g} / \mathrm{ml}$ streptomycin (both from Gibco; Thermo Fisher Scientific, Inc.) at $37^{\circ} \mathrm{C}$ in a humidified atmosphere containing $5 \% \mathrm{CO}_{2}$.

MTT assay. A total of $6 \times 10^{4}$ cells were seeded into a 96-well plate and pre-cultured with DMEM for $24 \mathrm{~h}$, then treated with PPI $(0.4-3 \mu \mathrm{M})$ for $24 \mathrm{~h}$ at $37^{\circ} \mathrm{C}$ in a humidified atmosphere containing $5 \% \mathrm{CO}_{2}$. Cell cytotoxicity was determined using MTT assay. The absorbance was measured at $490 \mathrm{~nm}$ using an automated microplate reader (BioTek Instruments, Inc., Winooski, VT, USA), and the inhibition rate was calculated as follows: Inhibition rate $(\%)=$ (average $\mathrm{A}_{490}$ of the control group-average $A_{490}$ of the experimental group)/(average $A_{490}$ of the control group-average $\mathrm{A}_{490}$ of the blank group) $\mathrm{x} 100 \%$. Cell viability was estimated using trypan blue dye exclusion.

Soft agar colony formation assay. A total of $1 \times 10^{3}$ cells were suspended in $1 \mathrm{ml}$ of DMEM containing $0.3 \%$ agarose (Amresco, Cleveland, OH, USA) and 10\% FBS, and plated on a bottom layer containing $0.6 \%$ agarose and $10 \%$ FBS in each of the triplicate wells of a 6 -well plate. Colonies formed on the soft agar plates were stained with $0.2 \%$ crystal violet and counted under light microscope (IX70; Olympus Corporation, Tokyo, Japan) after 2 weeks.

Invasion assay. Invasion assays were performed using a 24-well Transwell chamber (Corning Incorporated, Corning, NY, USA). Polyvinylpyrrolidone-free polycarbonate filters ( $8 \mu \mathrm{m}$ pore size) (Corning Incorporated) were coated with Matrigel (BD Biosciences, Franklin Lakes, NJ, USA). The lower chamber contained medium supplemented with
$20 \%$ FBS. Cells ( $1 \times 10^{4}$ cells/well) were placed into the upper chamber with the coated membrane. After incubation for $20 \mathrm{~h}$ at $37^{\circ} \mathrm{C}$, the invaded cells at the bottom of the membrane were fixed and stained with $2 \%$ ethanol containing $0.2 \%$ crystal violet (15 min) at room temperature for $20 \mathrm{~min}$ and observed under light microscope (x10 objective). For quantification, the invaded stained cells on the other side of the membrane were extracted with $33 \%$ acetic acid. The absorbance of the eluted stain was determined at $570 \mathrm{~nm}$.

Wound healing assay. Cells $\left(4 \times 10^{5}\right.$ cells $\left./ 2 \mathrm{ml}\right)$ were seeded in a 6-well plate and incubated at $37^{\circ} \mathrm{C}$ until they reach $90-100 \%$ confluence. Confluent cells were scratched using a $200 \mu \mathrm{l}$ pipet tip. After washing with PBS, cells were treated with serumfree medium for $24 \mathrm{~h}$. Then, the cells were fixed and stained with $2 \%$ ethanol containing $0.2 \%$ crystal violet powder at room temperature for $20 \mathrm{~min}$.

Reverse transcription quantitative polymerase chain reaction (RT-qPCR). Total RNA was extracted from cells using TRIzol reagent (Thermo Fisher Scientific, Inc.), according to the manufacturer's protocol. A total of $2 \mu \mathrm{g}$ RNA was reversetranscribed into cDNA using ReverTra Ace qPCR RT Kit (Toyobo Life Science, Osaka, Japan), according to the manufacturer's protocol. The reverse transcription steps as follow: $37^{\circ} \mathrm{C}$ for $15 \mathrm{~min}$ and $98^{\circ} \mathrm{C}$ for $5 \mathrm{~min}$. The samples were stored at $-20^{\circ} \mathrm{C}$. qPCR was performed using the SYBR ${ }^{\circledR}$ Green RealTime PCR Master Mix (Toyobo Life Science) and the ABI StepOnePlus ${ }^{\mathrm{TM}}$ Real-Time PCR System (ABI; Thermo Fisher Scientifc, Inc.), according to the manufacturer's protocol. GAPDH was used as an endogenous control. The PCR thermocycling conditions were as follows: Initial denaturation at $95^{\circ} \mathrm{C}$ for 3 min followed by 40 cycles of $95^{\circ} \mathrm{C}$ for $15 \mathrm{sec}$ and extension at $60^{\circ} \mathrm{C}$ for $1 \mathrm{~min}$. The threshold cycle for each sample was selected from the linear range and converted to a starting quantity by interpolation from a standard curve generated on the same plate for each set of primers (Table I). Epithelial (E)-cadherin, vimentin, matrix metalloproteinase 7 (MMP7), $M M P 9$ and $C I P 2 A$ mRNA levels were normalized for each well to GAPDH mRNA levels using the $2^{-\triangle \Delta C q}$ method (21). Each experiment was repeated three times.

Western blot analysis. Cells were lysed in radioimmunoprecipitation assay buffer containing $50 \mathrm{mM}$ Tris, $\mathrm{pH} 8.0,150 \mathrm{mM}$ $\mathrm{NaCl}, 0.1 \%$ SDS, $0.5 \%$ sodium deoxycholate, $1 \% \mathrm{NP}-40,1 \mathrm{mM}$ DTT, $1 \mathrm{mM} \mathrm{NaF}, 1 \mathrm{mM}$ sodium orthovanadate, $1 \mathrm{mM}$ phenylmethylsulfonyl fluoride (PMSF; Sigma-Aldrich; Merck KGaA) and $1 \%$ protease inhibitors cocktail (EMD Millipore, Billerica, MA, USA). Protein concentration was determined using the Bradford method. Equal amounts of sample $(25 \mu \mathrm{g})$ were separated by SDS-PAGE (8-12\% gels). Electrophoresed proteins were then transferred onto polyvinylidene fluoride membranes (EMD Millipore). The membranes were blocked with 5\% skimmed milk in Tris-buffered saline at room temperature for $1 \mathrm{~h}$. Following blocking, membranes were incubated overnight at $4^{\circ} \mathrm{C}$ with primary antibodies and then rinsed with Tris-buffered saline with Tween-20. The following primary antibodies were used: anti-E-cadherin $(1: 2,000$; 20874-1-AP), anti-vimentin (1:5,000; 10366-1-AP), anti-Snail (1:3,000; 13099-1-AP), anti-MMP7 (1:1,000; 10374-2-AP), 
anti-insulin-like growth factor-binding protein 3 (IGFBP3; 1:1,000; 10189-2-AP), anti-proliferating cell nuclear antigen (PCNA; 1:5,000; 10205-2-AP) (both from ProteinTech Group, Inc., Chicago, IL, USA); anti-MMP9 (1:500; sc-6840), anti-CIP2A (1:500; sc-80662); anti-Fos-related antigen 1 (FRA-1; 1:500; sc-271657) (Santa Cruz Biotechnology, Santa Cruz, CA, USA), anti-PP2A (1:1,000; 2038), anti-ERK1/2 (1:1,000; 9102), anti-phospho-ERK1/2 (Thr202/Tyr204) (1:1,000; 9101), anti-cFos (1:1,000; 2250; Cell Signaling Technology, Inc., Danvers, MA, USA), and anti-GAPDH (1:5,000; M20006; Abmart, Shanghai, China). Membranes were then washed, and incubated with horseradish peroxidase-conjugated secondary antibody (1:1,000; E030120-01 and E030110-01; EarthOx, LLC, San Francisco, CA, USA) for $1.5 \mathrm{~h}$ at room temperature. The protein bands were visualized using SuperSignal ${ }^{\circledast}$ West Pico PLUS Chemiluminescent substrate (34579; Pierce; Thermo Fisher Scientific, Inc.) (22). Densitometric quantification of the western blots was determined using ImageJ software (version 1.4.3.67; National Institutes of Health, Bethesda, MD, USA).

Luciferase reporter assay. DU145 and PC 3 cells were seeded in 12 -well culture plates. When cells reached $70 \%$ confluence, they were transfected with pAP-1-Luc plasmids (Beyotime Institute of Biotechnology, Haimen, China) using Lipofectamine 3000 (Thermo Fisher Scientifc, Inc.), according to manufacturer's protocol. Following transfection for $4 \mathrm{~h}$, the cells were treated with PPI (0.6-1.8 $\mu \mathrm{M})$ for $20 \mathrm{~h}$. Firefly luciferase activities were assayed using the Luciferase Assay System (Promega Biotech Co., Ltd., Beijing, China) according to the manufacturer's protocol.

Chromatin immunoprecipitation (ChIP) assay. DU145 and PC3 cells $\left(2 \times 10^{5}\right.$ cells $\left./ \mathrm{ml}\right)$ treated with PPI for $24 \mathrm{~h}$ were used for ChIP assay using a ChIP-IT ${ }^{\circledR}$ Express Enzymatic Shearing kit (Active Motif, Beijing, China), according to the manufacturer's protocol. DNA-bound protein was immunoprecipitated using an anti-c-Fos (component of AP-1) antibody or rabbit IgG as a control. The AP-1 binding site of MMP7 promoter was detected by RT-qPCR using the following primers: 5'-CCAGATGTTG CAGAATACTCACTA-3' (forward) and 5'-GATCCACTGT AATATGCGGTAAGT-3' (reverse). The AP-1 binding site of vimentin promoter was detected by RT-qPCR using the following primers: 5'-CGAGCTGGGCAGTAGAGAGT-3' (forward) and 5'-CCAAGACATGGAGGGAGTGT-3' (reverse).

PP2A activity assay. PP2A immunoprecipitation phosphatase assay kit (Upstate Biotechnology, Inc., Lake Placid, NY, USA) was used according to the manufacturer's protocol in order to evaluate phosphate release as an index of phosphatase activity. Briefly, $100 \mu \mathrm{g}$ protein isolated from cells was incubated with $4 \mu \mathrm{g}$ anti-PP2A monoclonal antibody overnight. A total of $4 \mu \mathrm{l}$ protein $\mathrm{A}$ agarose beads were added and the mixture was incubated at $4^{\circ} \mathrm{C}$ for $2 \mathrm{~h}$. Subsequently, the beads were collected and washed three times with $700 \mu 1$ ice-cold TBS and one time with $500 \mu 1 \mathrm{Ser} / \mathrm{Thr}$ assay buffer. The beads were further incubated with $750 \mathrm{mM}$ phosphopeptide in Ser/Thr assay buffer for $10 \mathrm{~min}$ at $30^{\circ} \mathrm{C}$ with constant agitation. A total of $100 \mu 1$ Malachite Green Phosphate Detection solution was added and the absorbance at $650 \mathrm{~nm}$ was measured using a microplate reader.
Table I. Primer sequences for reverse transcription quantitative polymerase chain reaction.

\begin{tabular}{ll}
\hline Gene & \multicolumn{1}{c}{ Primer sequence (5'-3') } \\
\hline E-cadherin & F: CAGGTCTCCTCATGGCTTTGC \\
& R: CTTCCGAAAAGAAGGCTGTCC \\
Vimentin & F: ACTACGTCCACCCGCACCTA \\
& R: CAGCGAGAAGTCCACCGAGT \\
$M M P 7$ & F: GAGGCATGAGTGAGCTACAGTG \\
& R: ACATCTGGGCTTCTGCATTATT \\
$M M P 9$ & F: CCGGACCAAGGATACAGTT \\
& R: CGGCACTGAGGAATGATCTA \\
CIP2A & F: TGCGGCACTTGGAGGTAATTTC \\
& R: AGCTCTACAAGGCAACTCAAGC \\
GAPDH & F: TGTTGCCATCAATGACCCTT \\
& R: CTCCACGACGTACTCAGCG
\end{tabular}

F, forward; R, reverse; E-cadherin, epithelial cadherin; MMP, matrix metalloproteinase; CIP2A, cancerous inhibitor of protein phosphatase $2 \mathrm{~A}$

Xenograft studies. Male nude immunodeficient mice (nu/nu) (weighing $\sim 16 \mathrm{~g}, 5$-week-old) were purchased from Hunan SJA Laboratory Animal Co., Ltd. (Changsha, China), and maintained under specific pathogen-free conditions. Mice were given free access to sterile water and to a standard diet, and maintained under controlled conditions of temperature $\left(22-24^{\circ} \mathrm{C}\right)$, humidity $(40-70 \%)$, light (12-h light-dark cycle). The present study was approved by the Hubei University of Medicine Animal Care and Use Committee (Hubei, China) and performed in compliance with the Regulations for the Administration of Affairs Concerning Experimental Animals. The mice were subcutaneously injected with human PC DU145 cells $\left(6 \times 10^{6}\right.$ cells which were suspended in $100 \mu \mathrm{l}$ DMEM media) into the right flank of each mouse. Treatments started when tumors reached a palpable size $(0.5 \mathrm{~cm}$ in diameter). Mice were randomly divided into the following two groups: control group (vehicle; $0.8 \%$ DMSO, $12 \%$ cremophor and $8 \%$ ethanol in normal saline; $\mathrm{n}=8$ ) and PPI-treated group (intraperitoneal injection of $1 \mathrm{mg} / \mathrm{kg} \mathrm{PP} 1 ; \mathrm{n}=8$ ). The mice were treated 5 times per week for a total of 30 weeks. Caliper measurements of the longest perpendicular tumor diameters were performed twice a week to estimate the tumor volume, using the following formula: $4 \pi / 3 \times(\text { width } / 2)^{2} \times($ length $/ 2)$, representing the 3 -dimensional volume of an ellipse. Animals were sacrificed when tumors reached $1.5 \mathrm{~cm}$ or if the mice appeared moribund to prevent unnecessary morbidity to the mice.

Statistical analysis. All experiments were repeated at least three times and the data are presented as the mean \pm standard deviation (SD). All statistical analyses were conducted using GraphPad Prism5 (GraphPad Software, Inc., La Jolla, CA, USA) and SPSS 22.0 (IBM Corp., Armonk, NY, USA). Results were analyzed using unpaired Student's t-test or one-way 


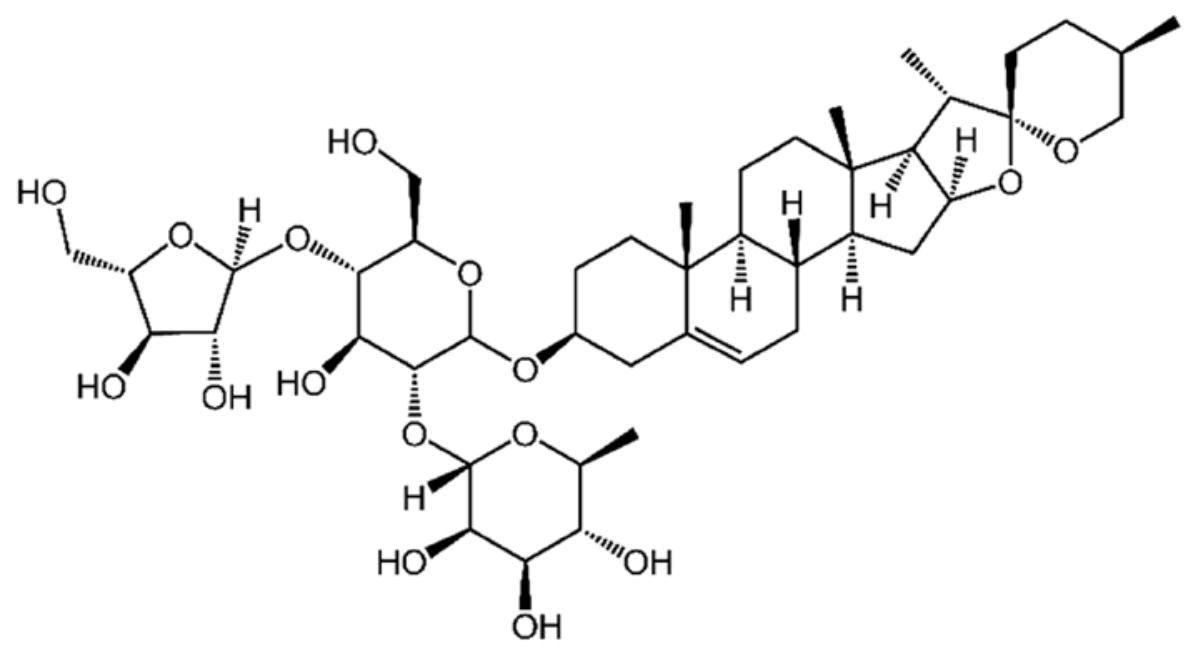

Figure 1. Chemical structure of Polyphyllin I.

A

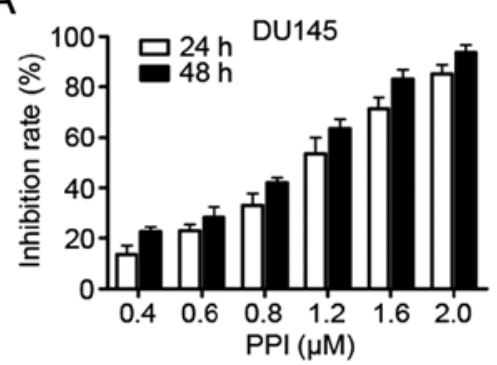

D

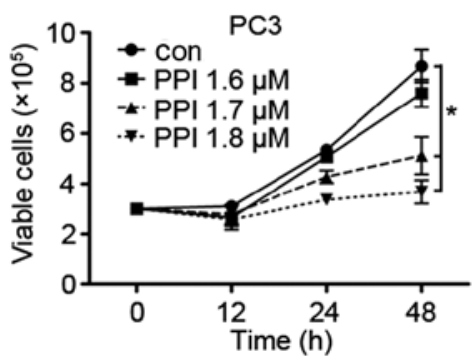

B

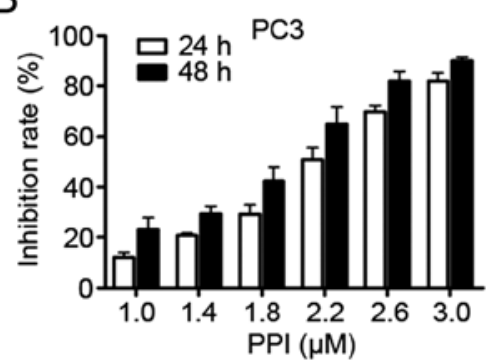

E

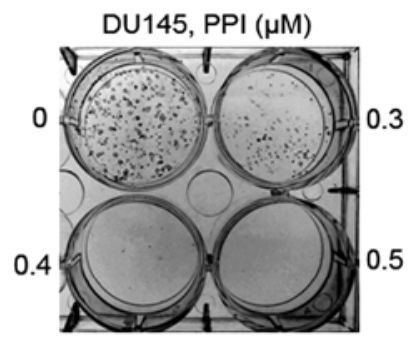

C

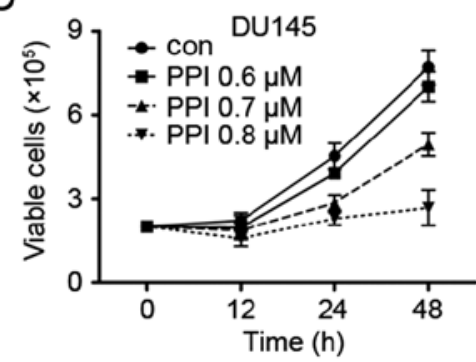

$\mathrm{F}$

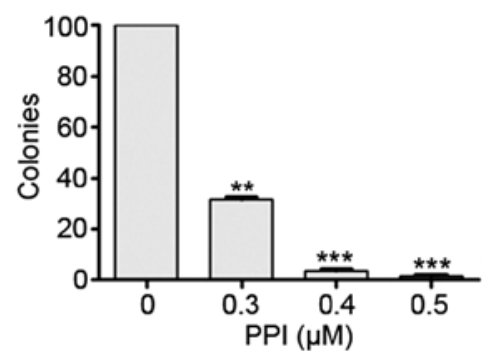

Figure 2. Effect of PPI treatment on viability and colony formation of DU145 and PC3 cells. MTT assay was used to examine the inhibitory effects of PPI treatment on (A) DU145 and (B) PC3 cells. Trypan blue exclusion assay was used to test the inhibitory effects of PPI treatment on cell viability of (C) DU145 and (D) PC3 cells. (E) Images display the colonies stained with crystal violet. (F) The graph represents the number of colonies of DU145 cells treated with PPI. ${ }^{* *} \mathrm{P}<0.01$ and ${ }^{* * *} \mathrm{P}<0.001$ vs. $0 \mu \mathrm{M}$. PPI, polyphyllin I; con, control.

analysis of variance followed by Bonferroni post-test. $\mathrm{P}<0.05$ was considered to indicate a statistically significant difference. All experiments were repeated at least three times.

\section{Results}

Effects of PPI on the viability of PC cell lines. DU145 and PC3 cells were seeded into 96 -well plates for $24 \mathrm{~h}$ and then treated with PPI (Fig. 1). After 24 or $48 \mathrm{~h}$, the cell survival rate was detected using the MTT assay. The results suggested that PPI exhibited moderate cytotoxicity to PC cells. The $\mathrm{IC}_{50}$ values of PPI against DU145 and PC 3 cells were $1.03 \mu \mathrm{M}$ and $2.13 \mu \mathrm{M}$, respectively (Fig. 2A and B). Trypan blue exclusion assay showed that PPI decreased viable DU145 and PC3 cells in a dosage- and time-dependent manner (Fig. 2C and D). Next, colony formation assay showed that PPI markedly suppressed the clonogenic ability of DU145 cells (Fig. 2E and F). These results suggest that PPI may decrease anchorage-dependent (cell viability) and anchorage-independent (colony formation) growth of PC cells.

PPI suppresses the invasion and migration of PC cells as well as reverses EMT. In the present study the effects of PPI on the invasion and migration of PCs (DU145 and PC3) cells were investigated. The results demonstrated that PPI suppressed the invasion of DU145 and PC3 cells (Fig. 3A). Additionally, cell migration was assessed using a wound healing assay. DU145 and PC 3 cells were treated with PPI for $24 \mathrm{~h}$. The results 
A
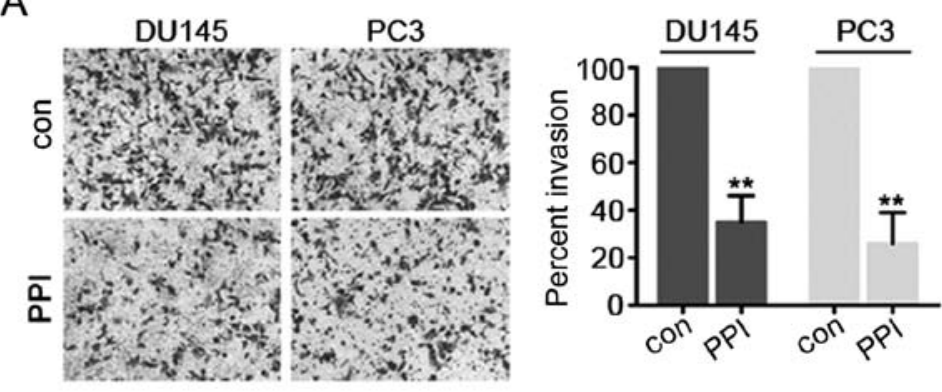

C

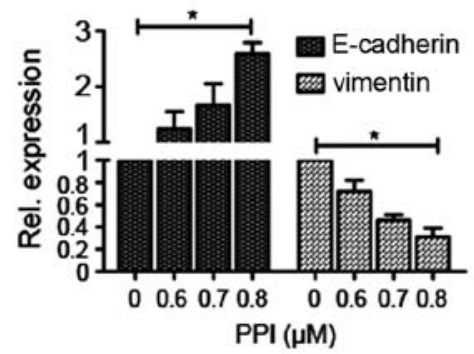

D

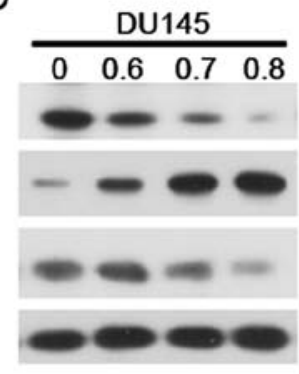

B

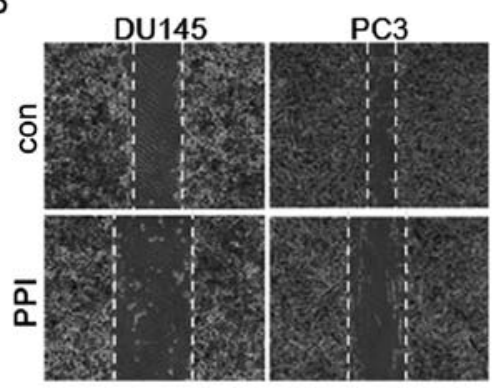

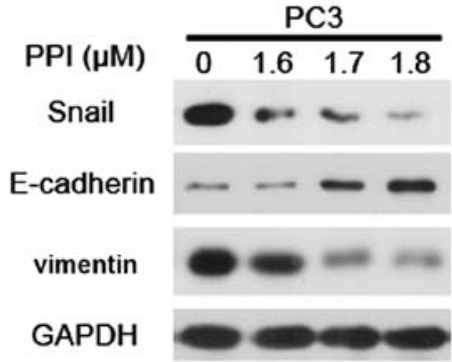

Figure 3. PPI inhibits invasive behavior and epithelial-mesenchymal transition of DU145 and PC3 cells. (A) Cells were pretreated with PPI (DU145 cells, $0.5 \mu \mathrm{M}$; PC3 cells $1.5 \mu \mathrm{M}$ ) for $30 \mathrm{~min}$. Invasion assay was performed using modified 24 -well microchemotaxis chambers (magnification, $\mathrm{x} 100)$. ${ }^{* *} \mathrm{P}<0.01 \mathrm{vs}$. con. (B) Confluent cells were scratched and then treated with PPI (DU145 cells, $0.5 \mu \mathrm{M}$; PC3 cells, $1.5 \mu \mathrm{M}$ ) for $24 \mathrm{~h}$. Migrated cells were examines (magnification, $\mathrm{x} 40)$. (C) Relative expression of E-cadherin and vimentin using reverse transcription quantitative polymerase chain reaction in DU145 and PC3 cells. ${ }^{*} \mathrm{P}<0.05$. (D) DU145 and PC3 cells were treated with various concentrations of PPI for 24 h. Western blot analysis were carried out using the indicated antibodies. PPI, polyphyllin I; con, control; E-cadherin, epithelial cadherin.

revealed that PPI decreased DU145 and PC3 cell migration (Fig. 3B). These results suggest that PPI may exhibit an anti-invasive effect on PCs at low concentrations.

EMT is an important process in tumor metastasis. During EMT, cells lose their polarized immotile epithelial characteristics and acquire motile mesenchymal features leading to increased invasion and motility (23). Moreover, the transition is characterized by a decrease in the expression of epithelial markers (including E-cadherin) as well as an increase in the expression of mesenchymal markers (including Snail and vimentin) $(23,24)$. To determine whether PPI treatment may reverse EMT and inhibit PC metastasis in vitro, the expression of EMT-associated markers were assessed in PPI-treated DU145 and PC3 cells. The results demonstrated an increased expression of E-cadherin and a decreased expression of vimentin in PPI-treated DU145 and PC3 cells compared with the control cells (Fig. 3C and D). These results indicate that PPI treatment may suppress EMT. The effects of PPI treatment on EMT-associated transcription factors were investigated and it was revealed that PPI treatment decreased the expression levels of Snail (Fig. 3C and D). Therefore, these results suggest that PPI upregulates E-cadherin expression, and downregulates the expression of Snail and vimentin.

PPI inhibits the invasion and EMT in PC cells by downregulating $M M P 7$, vimentin and Snail via the ERK signaling pathway. Tumor invasion and migration are associated with increased expression levels of matrix MMPs. MMP7 and MMP9 are two critical enzymes which contribute to cell invasion and migration $(25,26)$. Thus, $m R N A$ and protein expression levels of MMP7 and MMP9 were assessed (Fig. 4A-D). PPI treatment decreased MMP7 expression but it had no effect on MMP9 expression in DU145 and PC3 cells, suggesting that PPI may inhibit the invasion and migration of PC cells, by inhibiting MMP7.

Expression of MMP7, vimentin, and Snail may be regulated by the ERK signaling pathway, which serves an important role in invasion and EMT of PC cells (26-28). Therefore, the activation of ERK in PC cells treated with various PPI concentrations was assessed. The results demonstrated that PPI decreased the phosphorylation levels of ERK in a dose-dependent manner without affecting the total ERK expression and Akt phosphorylation in DU145 and PC3 cells (Fig. 4E and F). AP-1, a key transcription factor that promotes MMP7 and vimentin transcription, is regulated by MAPKs (including ERK, JNK and P38 kinases) $(27,29)$. Luciferase reporter assay was performed to investigate the effect of PPI on the transcriptional activity of AP-1 and the results indicated that PPI decreased AP-1 transcription activity (Fig. 4G). ChIP assays showed that PPI decreased c-Fos binding to the MMP7 promoter (Fig. $4 \mathrm{H}$ ). Additionally, PPI decreased FRA-1 (component of AP-1) binding to the vimentin promoter (Fig. 4I). These results demonstrate that PPI may inhibit invasion and EMT in PC cells through the ERK/AP-1 signaling pathway.

Effects of PPI treatment on the CIP2A/PP2A/ERK signaling axis. CIP2A is an oncogenic PP2A inhibitor, which is highly expressed in PC cells and mediates PC progression and castration-resistance (30). Western blot analysis was used to evaluate the effects PPI treatment on CIP2A protein expression. Fig. 5A shows that the expression of CIP2A was decreased in DU145 cells treated with $0.7 \mu \mathrm{M}$ PPI and could 
A

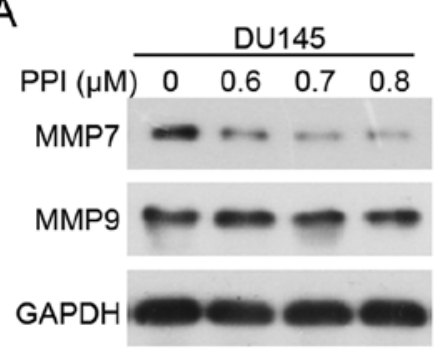

D

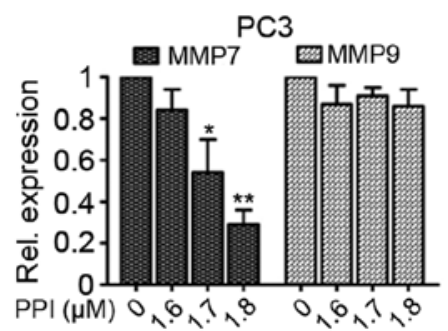

G

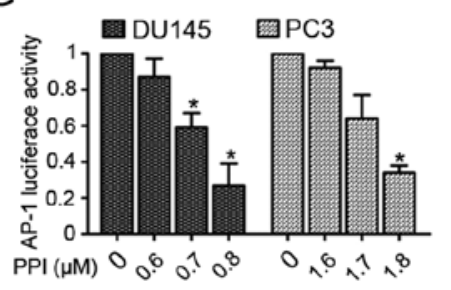

B

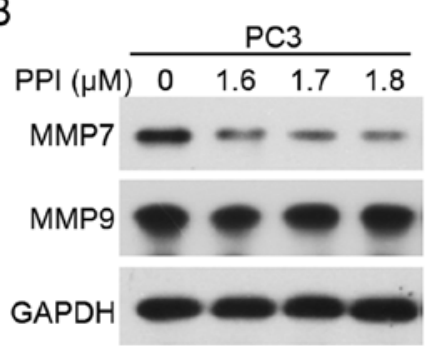

E

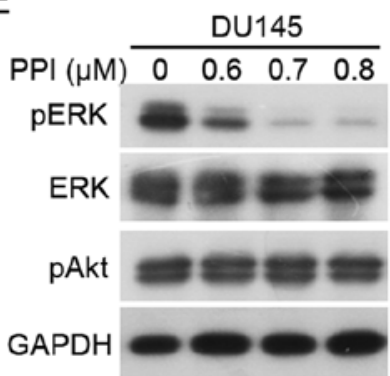

$\mathrm{H}$

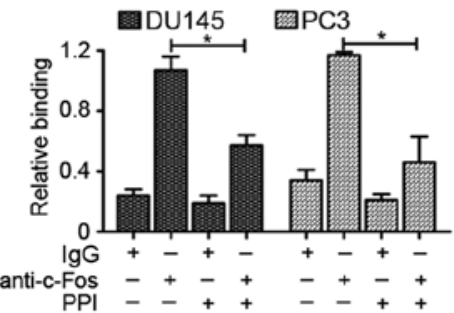

C

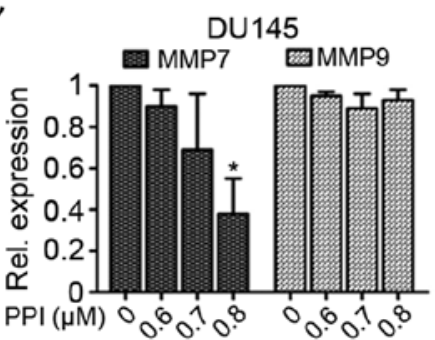

$\mathrm{F}$

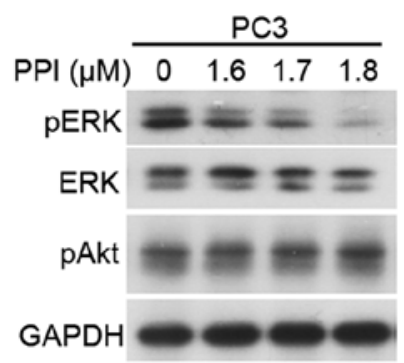

I

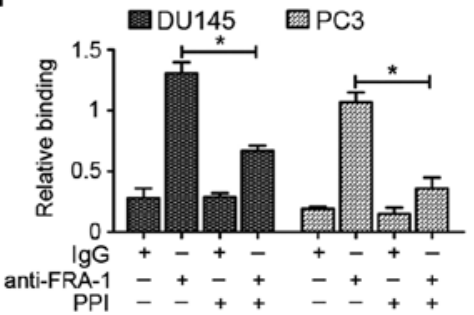

Figure 4. PPI suppresses PC cell invasion through the downregulation of MMP7 via ERK/AP-1 pathway. Western blot analysis for MMP7 and MMP9 expression in (A) DU145 and (B) PC3 cells were treated with PPI for 24 h. RT-qPCR analysis of mRNA levels of $M M P 7$ and $M M P 9$ in (C) DU145 and (D) PC3 cells treated with PPI for $24 \mathrm{~h}$. ${ }^{*} \mathrm{P}<0.05$ and ${ }^{* *} \mathrm{P}<0.01$ vs. $0 \mu \mathrm{M}$. Western blot analysis of pERK, ERK and pAkt expression in (E) DU145 and (F) PC3 cells were incubated with PPI at the indicated concentrations for $24 \mathrm{~h}$. (G) DU145 and PC3 cells were transfected with the AP-1 luciferase reporter construct for 4 h and then treated with PPI. Luciferase activity was measured using the luciferase assay system. ${ }^{*} \mathrm{P}<0.05 \mathrm{vs}$. $0 \mu \mathrm{M}$. (H) PPI inhibited binding of c-Fos to $M M P 7$ promoter. Chromatins were extracted from DU145 and PC3 cells treated with or without PPI (DU145 cells, $0.7 \mu \mathrm{M}$ PPI; PC3 cells, $1.7 \mu \mathrm{M}$ PPI). Chromatin immunoprecipitation was performed by using anti-c-Fos antibody, and the amplification was carried out by RT-qPCR. *P<0.05. (I) PPI inhibited binding of FRA-1 to vimentin promoter. Chromatins were extracted from DU145 and PC3 cells treated with or without PPI (DU145 cells, $0.7 \mu \mathrm{M}$ PPI; PC3 cells, $1.7 \mu$ M PPI). Chromatin immunoprecipitation was performed by using anti-FRA-1 antibody, and the amplification was carried out by RT-qPCR. RT-qPCR, reverse transcription quantitative polymerase chain reaction; PPI, polyphyllin I; ERK, extracellular signaling-related kinase; p, phosphorylated; MMP, matrix metalloproteinase; FRA, Fos-related antigen 1.

not be detected in response to $0.8 \mu \mathrm{M}$ PPI. Additionally, the expression of CIP2A was downregulated in response to treatment with $1.7 \mu \mathrm{M}$ PPI in PC3 cells, and treatment with $1.8 \mu \mathrm{M}$ PPI led to an elimination of CIP2A expression (Fig. 5B). In addition, the results indicated that PPI treatment may downregulates CIP2A expression in a time-dependent manner (Fig. 5C and D). The dephosphorylation of ERK is frequently regulated by $\mathrm{PP} 2 \mathrm{~A}$, which is the downstream factor of CIP2A, therefore whether PPI may affect PP2A activity was assessed. Fig. 5E showed that the activity of PP2A was increased in PPI-treated PC cells, indicating that PPI may influence the CIP2A/PP2A/ERK signaling axis. Moreover, a PP2A inhibitor, OA, was used for the in vitro experiments. The results indicated that OA reversed the cell growth and invasion that were inhibited by PPI treatment (Fig. 5F and G). Additionally, combined treatment with OA and PPI increased the expression levels of phosphorylated ERK and upregulated MMP7, Snail, vimentin expression compared with those in
PPI-treated cells (Fig. 5H and I). In conclusion, these results demonstrate that reactivation of $\mathrm{PP} 2 \mathrm{~A}$ may be required for PPI-inhibited invasion, MMP7, Snail, vimentin expression and ERK phosphorylation via CIP2A inhibition.

PPI suppresses tumor growth in vivo. To investigate the antitumor effect of PPI on PC in vivo, $6 \times 10^{6}$ DU145 cells in $100 \mu 1$ of DMEM medium were subcutaneously inoculated in the right flank of nude mice. The mice were divided into vehicle or PPI-treated groups. The results indicated that PPI treatment significantly suppressed tumor growth compared to the vehicle control $(\mathrm{P}<0.05$; Fig. $6 \mathrm{~A}$ and $\mathrm{B})$. PPI treatment also decreased the tumor weight of the mice (Fig. 6C). PPI treatment did not affect the body weight of the mice (Fig. 6D). Western blot analysis and PP2A activity assay revealed that the expression levels of pERK, MMP7, CIP2A and PCNA were decreased in the PPI-treated group (Fig. 6E). IGFBP3 is a critical biomarker involved in biological pathways thought 


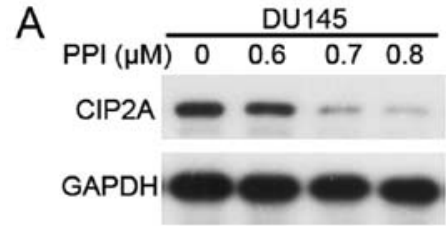

C

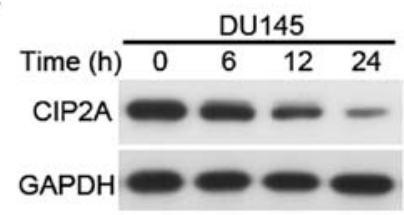

E

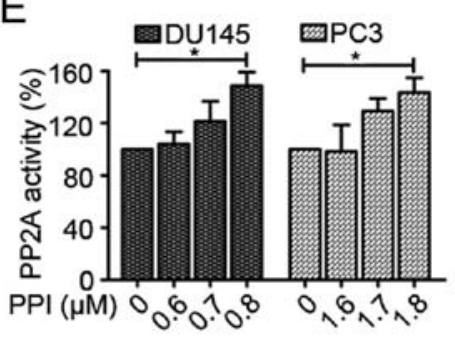

G

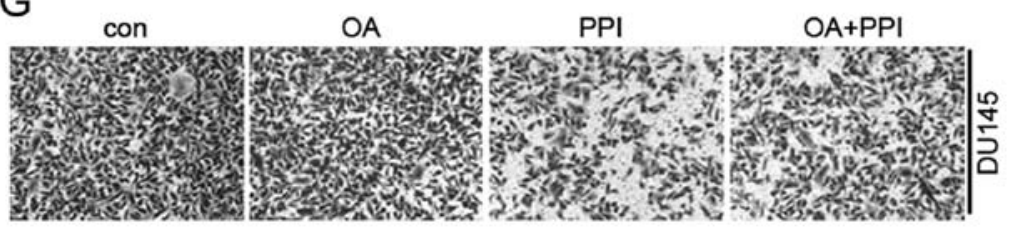

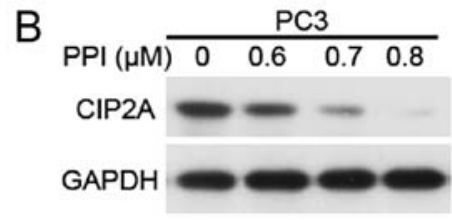

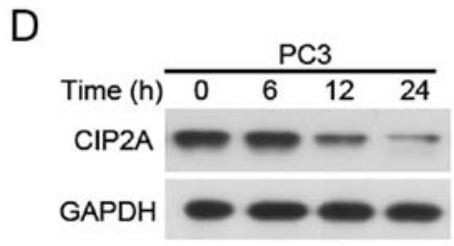

F
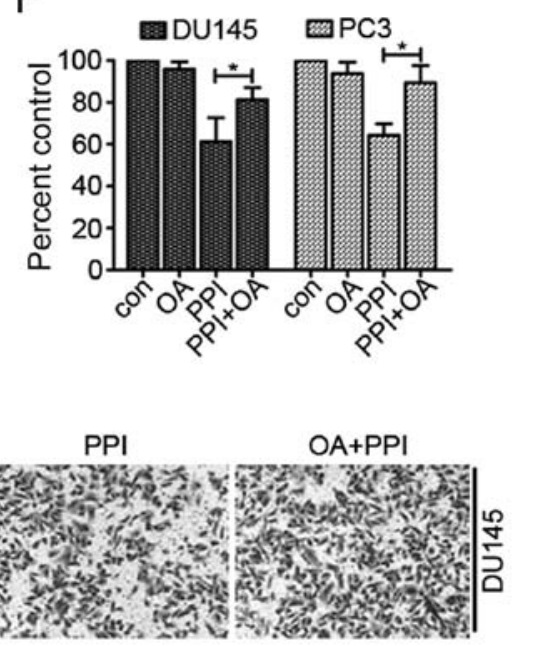
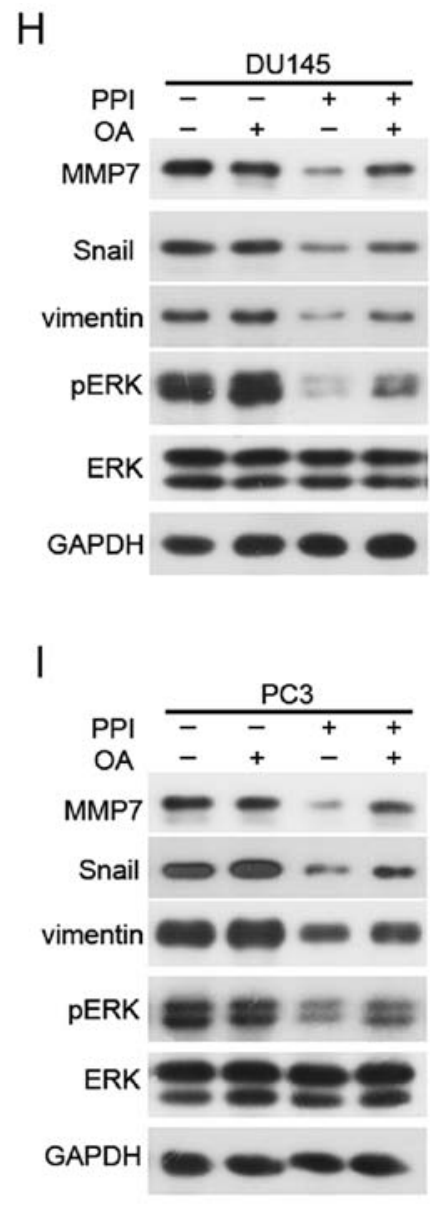

Figure 5. Effects of PPI on CIP2A/PP2A/ERK signaling axis. Western blot analysis of CIP2A expression in (A) DU145 and (B) PC3 cells treated with PPI for $24 \mathrm{~h}$. Western blot analysis of CIP2A expression in PPI-treated (C) DU145 and (D) PC3 cells for the indicated time-points. PP2A activity in (E) DU145 and PC3 cells treated with indicated concentrations of PPI for $24 .{ }^{*} \mathrm{P}<0.05$. (F) MMT assay in DU145 and PC3 cells were treated with PPI $(0.7$ or $1.7 \mu \mathrm{M})$ and/or OA $(50 \mathrm{nM})$ for 24 h. "P<0.05. (G) DU145 cells were treated with PPI $(0.6 \mu \mathrm{M})$ and/or OA $(50 \mathrm{nM})$ for $20 \mathrm{~h}$ and their invasive ability was examined. Western blot analysis of MMP7, Snail, vimentin, pERK and ERK in (H) DU145 and (I) PC3 cells treated with PPI and/or OA for 24 h. PPI, polyphyllin I; ERK, extracellular signaling-related kinase; p, phosphorylated; MMP, matrix metalloproteinase; CIP2A, cancerous inhibitor of protein phosphatase 2A; OA, okadaic acid; PP2A, protein phosphatase 2A.

to be important in pathogenesis and progression of PC (31). IGFBP3 expression was detected in tumor specimens and it was found that PPI decreased IGFBP3 expression (Fig. 6E). The PP2A activity of PPI-treated group was significantly upregulated (Fig. 6F).

Based on these results, PPI may suppress PC viability, invasion and EMT by inhibiting the CIP2A/PP2A/ERK signaling cascade (Fig. 7).

\section{Discussion}

$\mathrm{PC}$ is the second major cause of cancer mortality in males worldwide, which is due to late-stage diagnosis and inefficient therapy, especially for recurrent disease $(25,32)$. In Chinese traditional medicine, various compounds which are present in natural herbs may have an anticancer potential due to their ability to inhibit tumor growth, metastasis and angiogenesis without having significant side effects. Therefore, TCM may be an important approach for reducing the tumor incidence and mortality by preventing, reversing or delaying the process of tumorigenesis $(22,33,34)$. PPI is a compound found in Paris polyphylla rhizomes. PPI has been reported to exhibit antiproliferative and anticancer activities according to previous studies $(16,18,19)$. Therefore, the mode of action and potential utility of PPI was explored in PC cells in search of a novel, effective anticancer agent.

In the present study, the effects of PPI were investigated using DU145 and PC3 cell lines. MTT and trypan blue dye exclusion assays suggested that PPI may suppress the growth of PC cells. Furthermore, PPI may inhibit the colony formation ability of PC cells. Therefore, PPI was shown to exert anti-proliferative effects in human PC cells.

Inhibition of tumor metastasis and invasion is critical to improving the prognosis of patients with PC (26). Therefore, treatment strategies for prevention or suppression of tumor metastasis and invasion may ameliorate the survival of patients with PC. The results of the present study suggest that PPI may suppress the invasive and migratory abilities of DU145 and PC3 cells. Tumor metastasis is a multifactor, multistage and multistep process regulated by multiple factors and genes. EMT serves a key role in cancer metastasis (35). Therefore, in the present study the expression of EMT-associated markers was investigated and it was found that PPI-mediated mobility inhibition was associated with EMT reversion. 
A

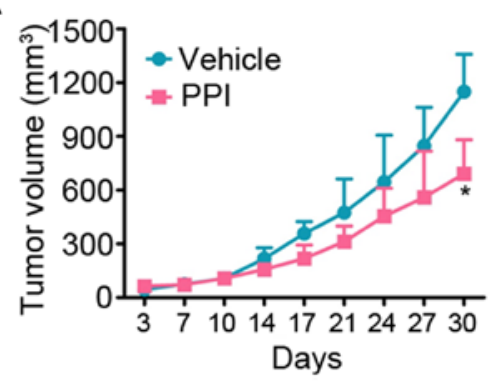

C

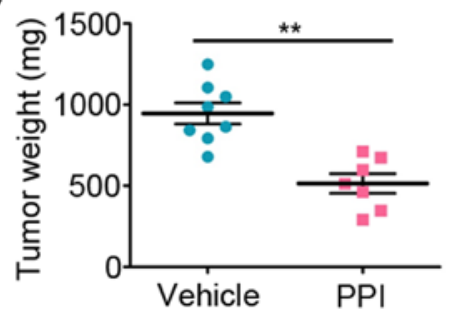

$E$

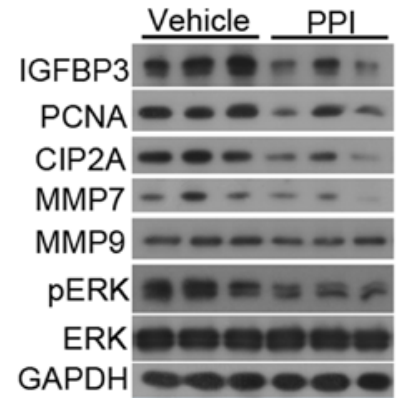

B

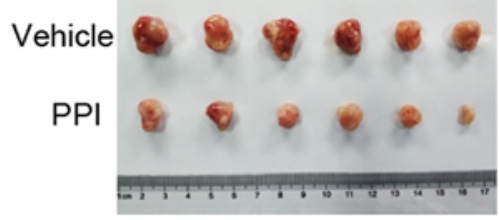

D

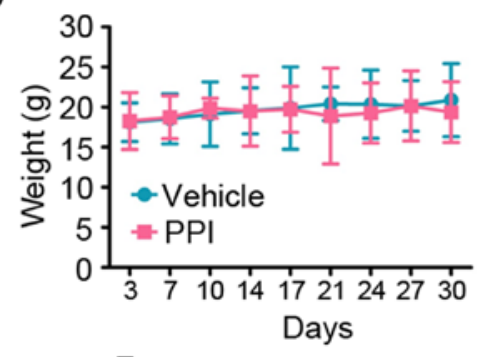

$\mathrm{F}$

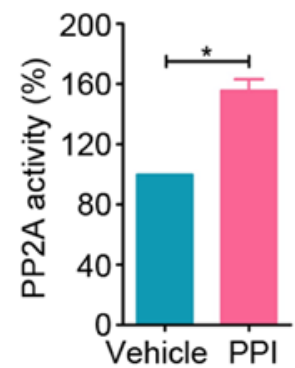

Figure 6. PPI suppresses tumor growth in a xenograft mouse model. (A) Mice were treated with vehicle or $1.5 \mathrm{mg} / \mathrm{kg}$ PPI and the tumor volumes were evaluated twice a week. "P<0.05 vs. vehicle. (B) Images of xenograft tumors separated from mice treated with vehicle or $1.5 \mathrm{mg} / \mathrm{kg}$ PPI after 4 weeks. (C) Weight of the tumor from vehicle or $1.5 \mathrm{mg} / \mathrm{kg}$ PPI-treated mice. ${ }^{* *} \mathrm{P}<0.01$ vs. vehicle. (D) Body weight of the vehicle or $1.5 \mathrm{mg} / \mathrm{kg}$ PPI-treated mice. (E) The expression of indicated proteins in xenograft tumors was detected using western blot analysis. (F) Tumor tissues were isolated from mice, and PP2A activity was detected using PP2A phosphatase assay. "P<0.05. PPI, polyphyllin I; ERK, extracellular signaling-related kinase; p, phosphorylated; MMP, matrix metalloproteinase; CIP2A, cancerous inhibitor of protein phosphatase 2A; PP2A, protein phosphatase 2A; IGFBP3, insulin-like growth factor-binding protein 3; PCNA, proliferating cell nuclear antigen.

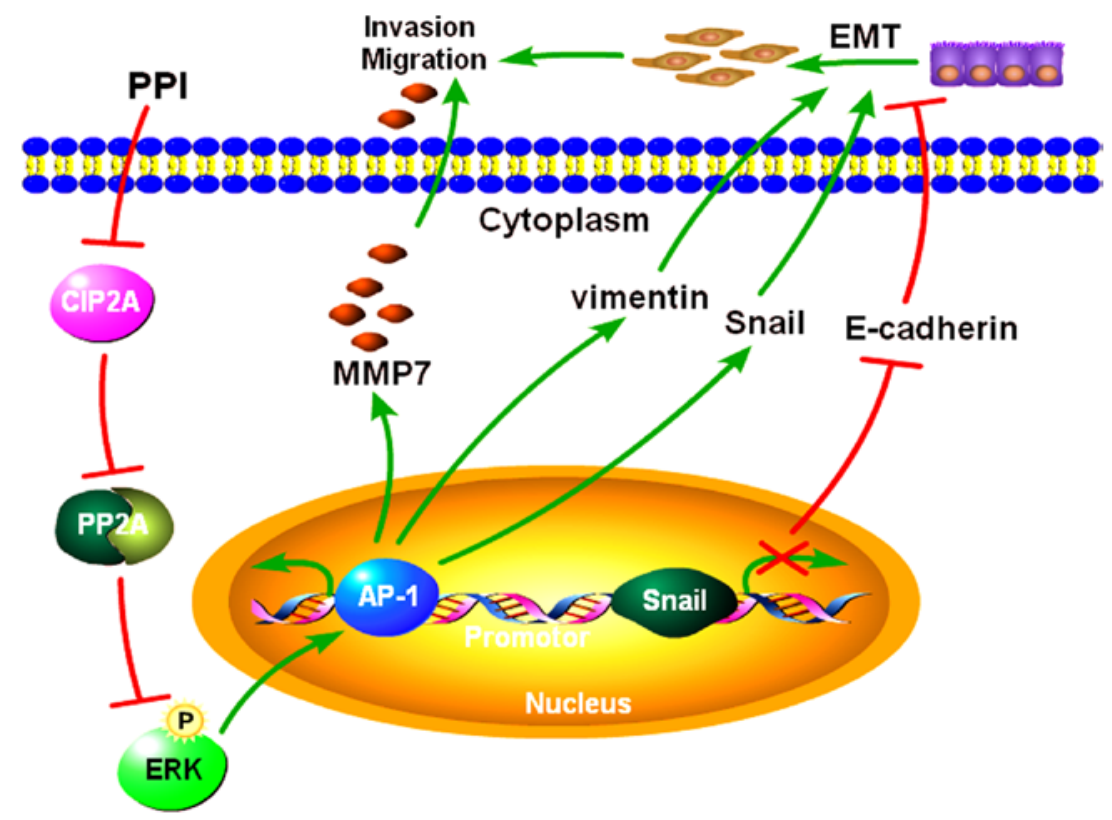

Figure 7. Diagram of PPI blockage possible mechanism in prostate cancer cells. PPI, polyphyllin I; ERK, extracellular signaling-related kinase; p, phosphorylated; MMP, matrix metalloproteinase; CIP2A, cancerous inhibitor of protein phosphatase 2A; PP2A, protein phosphatase 2A; EMT, epithelial-mesenchymal transition; E-cadherin, epithelial cadherin. 
Another finding of the present study was that PPI may inhibit the expression of MMP7. Metastasis and invasion of tumor cells involves the following processes: adhesion, enzymolysis and movement. MMP7 is a membrane protein in the MMP family (29). This type of protease degrades the extracellular matrix and basement membrane, which are important mediators for the metastasis and invasion of cancer cells (26). Previous studies have confirmed that transcription factors, including Snail, Slug and ZEB1, which act as E-cadherin repressors, are involved in promoting EMT (36). And MMP7, vimentin and Snail expression is regulated by the ERK signaling pathway (27-29). In the present study the effects of PPI on the phosphorylation level of ERK were examined. The results demonstrated that PPI may inhibit ERK phosphorylation in DU145 and PC3 cells. The transcription factor AP-1 may form either a homo- or hetero- complex which can bind as c-fos/c-fos, c-jun/c-jun or c-fos/c-jun to activate endogenous or exogenous motivation (37). AP-1 is also a critical transcriptional modulator in ERK signal transduction (15). Moreover, the MMP7 and vimentin promoter region contains a cisregulatory element of one AP-1 binding site. Thus, the effects of PPI on the AP-1 transcription activity and binding ability to the MMP7 or vimentin promoter was assessed. The results revealed that PPI may suppress AP-1 transcriptional activity and AP-1 binding ability to the $M M P 7$ and vimentin promoter. In conclusion, these results suggest that the anti-invasive effect of PPI may be mediated by suppressing AP-1 transcription activities and the ERK/MMP7 and ERK/EMT signal.

CIP2A is a oncogene that may facilitate cell transformation and tumorigenesis via inhibiting PP2A activities, as it is a protein phosphatase that regulates several critical oncogenic signal proteins including Akt, c-Myc, ERK and p70S6K (38-40). The results of the present study revealed that PPI may downregulate CIP2A expression and upregulate PP2A activity. It was speculated that PPI may inhibit CIP2A/PP2A signal to downregulate ERK phosphorylation and the expression of MMP7, vimentin, and Snail, and then decrease PC cell EMT and invasion. To further determine the effect of PP2A activation in PPI-mediated inhibition of invasion and downregulation of MMP7, PCs were treated with PPI and OA, an inhibitor of PP2A. The results showed that inactivation of PP2A may antagonize the effect of PPI in EMT and invasion through the ERK pathway. These results confirmed that the CIP2A/PP2A signaling axis serves a crucial function in PC proliferation, invasion, and EMT that is mediated by inhibiting ERK activity. ERK activation may be induced by PPI. In a xenograft mouse model, PPI significantly repressed tumor growth, whereas the body weight of the mice remained unaffected. The results also demonstrated that PPI suppressed pERK, MMP7, IGFBP3 and CIP2A expression. Therefore, PPI may be considered a novel anticancer agent in preventing and treating $\mathrm{PC}$.

\section{Acknowledgements}

Not applicable.

\section{Funding}

The present study was supported by the National Natural Sciences Foundation of China (grant nos. 81400157 and
11647002); The Foundation for Innovative Research Team of Hubei University of Medicine (grant no. FDFR201801); the Scientific and Technological Project of Shiyan City of Hubei Province (grant no. 17Y01); the Natural Science Foundation of Anhui Province of China (grant no. 1708085QH217); and the Foundation of Anhui Medical University (grant no. XJ201616).

\section{Availability of data and materials}

Materials described in the manuscript will be freely available to any scientist wishing to use them for non-commercial purposes without breaching participant confidentiality.

\section{Authors' contributions}

The project was conceived and designed by YT and YL. The experiments were conducted by XL, ZS, JD, JL, KM, YS and TF. Data were analyzed by XL and YL. The manuscript was written by YT and YL.

\section{Ethics approval and consent to participate}

All animal studies were conducted according to protocols approved by the Hubei University of Medicine Animal Care and Use Committee (approval no. 2017-175), complying with the Regulations for the Administration of Affairs Concerning Experimental Animals.

\section{Patient consent for publication}

Not applicable.

\section{Competing interests}

The authors declare that they have no competing interests.

\section{References}

1. Torre LA, Bray F, Siegel RL, Ferlay J, Lortet-Tieulent J and Jemal A: Global cancer statistics, 2012. CA Cancer J Clin 65: 87-108, 2015.

2. Chen W, Zheng R, Baade PD, Zhang S, Zeng H, Bray F, Jemal A, $\mathrm{Yu}$ XQ and He J: Cancer statistics in China, 2015. CA Cancer J Clin 66: 115-132, 2016.

3. Siegel RL, Miller KD and Jemal A: Cancer Statistics, 2017. CA Cancer J Clin 67: 7-30, 2017.

4. Junttila MR, Puustinen P, Niemelä M, Ahola R, Arnold H, Böttzauw T, Ala-aho R, Nielsen C, Ivaska J, Taya Y, et al: CIP2A inhibits PP2A in human malignancies. Cell 130: 51-62, 2007.

5. Khanna A, Böckelman C, Hemmes A, Junttila MR, Wiksten JP, Lundin M, Junnila S, Murphy DJ, Evan GI, Haglund C, et al: MYC-dependent regulation and prognostic role of CIP2A in gastric cancer. J Natl Cancer Inst 101: 793-805, 2009.

6. Teng HW, Yang SH, Lin JK, Chen WS, Lin TC, Jiang JK, Yen CC, $\mathrm{Li} \mathrm{AF}$, Chen PC, Lan YT, et al: CIP2A is a predictor of poor prognosis in colon cancer. J Gastrointest Surg 16: 1037-1047, 2012.

7. Vaarala MH, Väisänen MR and Ristimäki A: CIP2A expression is increased in prostate cancer. J Exp Clin Cancer Res 29: 136, 2010.

8. Böckelman C, Lassus H, Hemmes A, Leminen A, Westermarck J, Haglund C, Bützow R and Ristimäki A: Prognostic role of CIP2A expression in serous ovarian cancer. Br J Cancer 105: 989-995, 2011.

9. Tseng LM, Liu CY, Chang KC, Chu PY, Shiau CW and Chen KF: CIP2A is a target of bortezomib in human triple negative breast cancer cells. Breast Cancer Res 14: R68, 2012. 
10. Liu Z, Ma L, Wen ZS, Hu Z, Wu FQ, Li W, Liu J and Zhou GB Cancerous inhibitor of PP2A is targeted by natural compound celastrol for degradation in non-small-cell lung cancer. Carcinogenesis 35: 905-914, 2014

11. Côme C, Laine A, Chanrion M, Edgren H, Mattila E, Liu X, Jonkers J, Ivaska J, Isola J, Darbon JM, et al: CIP2A is associated with human breast cancer aggressivity. Clin Cancer Res 15: 5092-5100, 2009.

12. De P, Carlson JH, Leyland-Jones B and Dey N: Role of 'oncogenic nexus' of CIP2A in breast oncogenesis: How does it work? Am J Cancer Res 5: 2872-2891, 2015.

13. Wu Y, Gu TT and Zheng PS: CIP2A cooperates with H-Ras to promote epithelial-mesenchymal transition in cervical-cancer progression. Cancer Lett 356: 646-655, 2015.

14. Cai F, Zhang L, Xiao X, Duan C, Huang Q, Fan C, Li J, Liu X, Li S and Liu Y: Cucurbitacin B reverses multidrug resistance by targeting CIP2A to reactivate protein phosphatase $2 \mathrm{~A}$ in MCF-7/adriamycin cells. Oncol Rep 36: 1180-1186, 2016.

15. Xiao X, He Z, Cao W, Cai F, Zhang L, Huang Q, Fan C, Duan C, Wang X, Wang $\mathrm{J}$, et al: Oridonin inhibits gefitinib-resistant lung cancer cells by suppressing EGFR/ERK/MMP-12 and CIP2A/Akt signaling pathways. Int J Oncol 48: 2608-2618, 2016.

16. Li GB, Fu RQ, Shen HM, Zhou J, Hu XY, Liu YX, Li YN, Zhang HW, Liu X, Zhang YH, et al: Polyphyllin I induces mitophagic and apoptotic cell death in human breast cancer cells by increasing mitochondrial PINK1 levels. Oncotarget 8: 10359-10374, 2017.

17. Chen JC, Hsieh MJ, Chen CJ, Lin JT, Lo YS, Chuang YC, Chien SY and Chen MK: Polyphyllin G induce apoptosis and autophagy in human nasopharyngeal cancer cells by modulation of AKT and mitogen-activated protein kinase pathways in vitro and in vivo. Oncotarget 7: 70276-70289, 2016.

18. He H,Zheng L, Sun YP, Zhang GW and Yue ZG: Steroidal saponins from Paris polyphylla suppress adhesion, migration and invasion of human lung cancer A549 cells via down-regulating MMP-2 and MMP-9. Asian Pac J Cancer Prev 15: 10911-10916, 2014.

19. Yue G, Wei J, Qian X, Yu L, Zou Z, Guan W, Wang H, Shen J and Liu B: Synergistic anticancer effects of polyphyllin I and evodiamine on freshly-removed human gastric tumors. PLoS One 8: e65164, 2013.

20. Shi YM, Yang L, Geng YD, Zhang C and Kong LY: Polyphyllin I induced-apoptosis is enhanced by inhibition of autophagy in human hepatocellular carcinoma cells. Phytomedicine 22: $1139-1149,2015$

21. Livak KJ and Schmittgen TD: Analysis of relative gene expression data using real-time quantitative PCR and the 2(-Delta Delta C(T)) method. Methods 25: 402-408, 2001.

22. Cao W, Liu Y, Zhang R, Zhang B, Wang T, Zhu X, Mei L, Chen H, Zhang H, Ming P, et al: Homoharringtonine induces apoptosis and inhibits STAT3 via IL-6/JAK1/STAT3 signal pathway in Gefitinib-resistant lung cancer cells. Sci Rep 5: 8477, 2015.

23. Bucay N, Bhagirath D, Sekhon K, Yang T, Fukuhara S, Majid S, Shahryari V, Tabatabai Z, Greene KL, Hashimoto Y, et al: A novel microRNA regulator of prostate cancer epithelial-mesenchymal transition. Cell Death Differ 24: 1263-1274, 2017.

24. Montanari M, Rossetti S, Cavaliere C, D'Aniello C, Malzone MG, Vanacore D, Di Franco R, La Mantia E, Iovane G, Piscitelli R, et al: Epithelial-mesenchymal transition in prostate cancer: An overview. Oncotarget 8: 35376-35389, 2017.

25. Chen CM, Lin CL, Chiou HL, Hsieh SC, Lin CL, Cheng CW, Hung CH, Tsai JP and Hsieh YH: Loss of endothelial cell-specific molecule 1 promotes the tumorigenicity and metastasis of prostate cancer cells through regulation of the TIMP-1/MMP-9 expression. Oncotarget 8: 13886-13897, 2017.

26. Zhang Q, Liu S, Parajuli KR, Zhang W, Zhang K, Mo Z, Liu J, Chen Z, Yang S, Wang AR, et al: Interleukin-17 promotes prostate cancer via MMP7-induced epithelial-to-mesenchymal transition. Oncogene 36: 687-699, 2017.
27. Andreolas C, Kalogeropoulou M, Voulgari A and Pintzas A: Fra-1 regulates vimentin during Ha-RAS-induced epithelial mesenchymal transition in human colon carcinoma cells. Int J Cancer 122: 1745-1756, 2008.

28. Zhao J, Ou B, Han D, Wang P, Zong Y, Zhu C, Liu D, Zheng M, Sun J, Feng $\mathrm{H}$, et al: Tumor-derived CXCL5 promotes human colorectal cancer metastasis through activation of the ERK/Elk-1/Snail and AKT/GSK3 $/ / \beta$-catenin pathways. Mol Cancer 16: 70, 2017.

29. Chang MC, Chen CA, Chen PJ, Chiang YC, Chen YL, Mao TL, Lin HW, Lin Chiang WH and Cheng WF: Mesothelin enhances invasion of ovarian cancer by inducing MMP-7 through MAPK/ERK and JNK pathways. Biochem J 442: 293-302, 2012.

30. Khanna A, Rane JK, Kivinummi KK, Urbanucci A, Helenius MA, Tolonen TT, Saramäki OR, Latonen L, Manni V, Pimanda JE, et al: CIP2A is a candidate therapeutic target in clinically challenging prostate cancer cell populations. Oncotarget 6: 19661-19670, 2015.

31. Terracciano D, Bruzzese D, Ferro M, Mazzarella C, Di Lorenzo G, Altieri V, Mariano A, Macchia V and Di Carlo A: Preoperative insulin-like growth factor-binding protein-3 (IGFBP-3) blood level predicts gleason sum upgrading. Prostate 72: 100-107, 2012.

32. Terracciano D, Mazzarella C, Di Carlo A, Mariano A, Ferro M, Di Lorenzo G, Giordano A, Altieri V, De Placido S and Macchia V: Effects of the ErbB1/ErbB2 kinase inhibitor GW2974 on androgen-independent prostate cancer PC-3 cell line growth and NSE, chromogranin A and osteopontin content. Oncol Rep 24: 213-217, 2010.

33. Feng T, Cao W, Shen W, Zhang L, Gu X, Guo Y, Tsai HI, Liu X, Li J, Zhang J, et al: Arctigenin inhibits STAT3 and exhibits anticancer potential in human triple-negative breast cancer therapy. Oncotarget 8: 329-344, 2017.

34. Liu Y, Dong Y, Zhang B and Cheng YX: Small compound 6-O-angeloylplenolin induces caspase-dependent apoptosis in human multiple myeloma cells. Oncol Lett 6: 556-558, 2013.

35. Cao Z, Koochekpour S, Strup SE and Kyprianou N: Reversion of epithelial-mesenchymal transition by a novel agent DZ-50 via IGF binding protein-3 in prostate cancer cells. Oncotarget 8: 78507-78519, 2017.

36. Chen HN, Yuan K, Xie N, Wang K, Huang Z, Chen Y, Dou Q, Wu M, Nice EC, Zhou ZG, et al: PDLIM1 stabilizes the E-cadherin/ $\beta$-catenin complex to prevent epithelial-mesenchymal transition and metastatic potential of colorectal cancer cells. Cancer Res 76: 1122-1134, 2016.

37. Wen-Sheng W: ERK signaling pathway is involved in $\mathrm{p} 15 \mathrm{INK} 4 \mathrm{~b} /$ p16INK4a expression and HepG2 growth inhibition triggered by TPA and Saikosaponin a. Oncogene 22: 955-963, 2003.

38. Rincón R, Cristóbal I, Zazo S, Arpí O, Menéndez S, Manso R, Lluch A, Eroles P, Rovira A, Albanell J, et al: PP2A inhibition determines poor outcome and doxorubicin resistance in early breast cancer and its activation shows promising therapeutic effects. Oncotarget 6: 4299-4314, 2015.

39. Tsukamoto S, Huang Y, Umeda D, Yamada S, Yamashita S, Kumazoe M, Kim Y, Murata M, Yamada K and Tachibana H: $67-\mathrm{kDa}$ laminin receptor-dependent protein phosphatase $2 \mathrm{~A}$ (PP2A) activation elicits melanoma-specific antitumor activity overcoming drug resistance. J Biol Chem 289: 32671-32681, 2014.

40. Wang J, Okkeri J, Pavic K, Wang Z, Kauko O, Halonen T, Sarek G, Ojala PM, Rao Z, Xu W, et al: Oncoprotein CIP2A is stabilized via interaction with tumor suppressor PP2A/B56. EMBO Rep 18: 437-450, 2017. 